\title{
Twisted nose and anterior nasal valve plasty classification you can rely on
}

\section{Introduction and terminology}

The twisted nose pathophysiology is trauma, growth, iatrogenic and congenital; it has a cosmetic and functional implication. Both pathologies are usually connected.

The integrity of the internal anterior nasal valve is governed by the integrity of the upper alar cartilage junction with the nasal septal areas anterior to Cottle's line and by the anterior part of the inferior turbinate. It is the areas which represent the middle third of the external nose.

The external anterior nasal valve integrity is governed by the lower alar cartilage shape and the strength of its structures. The very caudal end of the nasal septum could play part if it is severely dislocated. The lower third of the external nose is the area of that part and its deformity could have an implication on the nasal valve patency.

The deviation on the lower two thirds of the external nose should raise a concern about the anterior nasal patency.

The upper third bony structures of the external nose is the main derive of the semi rigid cartilaginous and its cover SMAS superficial musculoaponeuratic system on its lower two thirds.

Three key stone points are vital for the nasal alignment and anterior nasal valve patency; The middle and lateral bony cartilaginous connection, where the upper alar cartilage tucks to the undersurface of the external nasal bones.

\section{Clinical assessment}

For the purpose of study, the external nose is divided to three parts; upper body part and two lower cartilaginous parts. The standard midline is the vertical line which connects the glabellar mid brows point to the lowest mid upper lip philtrum point. The brow tip line on each side identify the existent nasal alignment, the nose is straight where the midline cross equally between the two brow tip lines all the way through the three parts of the nose (Figure 1).

\section{Classification of nasal deviation}

I adopt the following system for quantifying the external nasal deviation on each of the three parts separately.

Grade 1 nasal deviation: the midline doesn't cross equally between the two brow tip lines but does not touch any of them.

Grade 2nasal deviation: the midline touches one of the brow tip lines but do not go beyond it.

Grade 3nasal deviation:the midline goes further lateral than one of the brow tip lines.

For example I say: the nose is deviated as following: upper left Grade 1, middle left Grade 2 and lower left Grade 3, it means the whole nose is twisted linearly and increasengly from the bony root down to the nasal tip on the left (Figure 1). Abdul Juratli
Consultant ENT Surgeon at LRI hospital University of Leicester,
UK

Correspondence: Abdul Juratli FRCS Consultant ENT Surgeon at LRI hospital University of Leicester Leicester, UK, Email juratli3@yahoo.co.uk

Received: April 23, 2017| Published: May 15, 2017

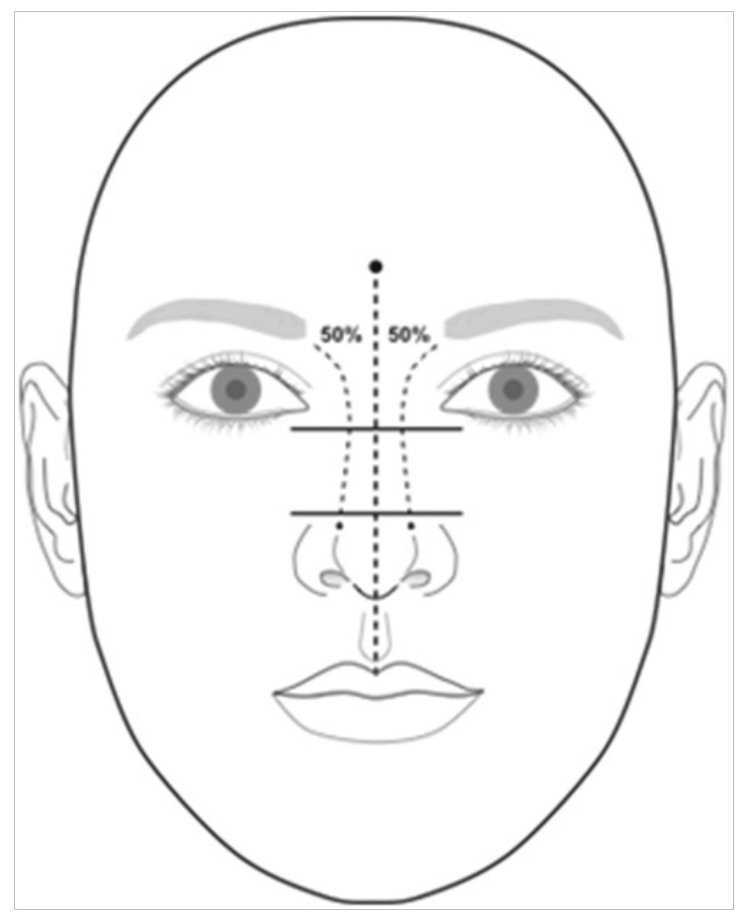

Figure I Classification of twisted nose.

\section{Classification of nasal septal areas}

The Nasal Septum is divided for a functional purpose to five areas. M.Cottle, Modified Cottle line is the straight line which connect the nasal process of the frontal bone to the maxillary crest one centimetre behind the anterior nasal spine. Areas $1,2 \& 3$ are located anterior to the line while area $4 \& 5$ are posteriorly located. The new M.Cottle areas classification gives clearer cut to the classical Cottle's classification of nasal septal areas (Figure 2) 


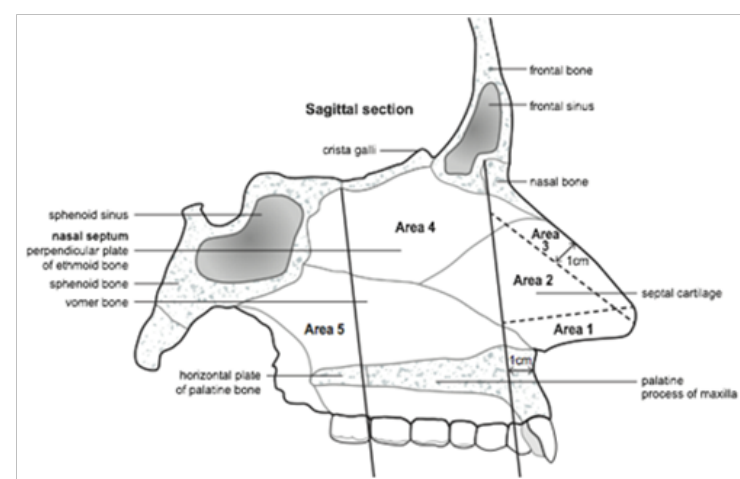

Figure 2 Modified Cottle's Septal areas \& Modified Cottle's Septal line.

Area 1: is the first one centimetre of the caudal septum parallel to the free caudal septal line. It is one $\mathrm{cm}$ cephalic to the anterior septal angle.

Area 2: is the one centimetre of the septal dorsal strip anterior to the M.Cottle line. It extends from the upper one $\mathrm{cm}$ of the M.Cottle line caudally parallel to the external nasal alignment down to the free septal caudal line.

Note: area one and two share the one $\mathrm{cm}$ square around the anterior septal angle.

Area 3: is the area between M.Cottle line and the inner boundaries of Areas $1 \& 3$

Area 4: is the area behind and parallel to M.Cottle line back to the level opposite to the inferior point of the middle turbinate ground lamella.

Area 5: It is the rest of the septum between the posterior line of area four and the posterior septal edge.

\section{Functional implication of the septal areas}

The first three areas are responsible for maintaining the anterior nasal valve function and the external nasal alignment, the one centimetre square shared area between $1 \& 3$ are the cornerstone septal area for stabilising the nasal tip on the middle, maintaining the tip projection and rotation. Area four deals with the osteomeatal hygiene and olfactory function, it gives an autogenous septal perpendicular plate graft which is frequently used by the author for maintaining the external nasal alignment. Areas five is responsible for maintaining the integrity of the posterior choanae and have a possible unidentified role on diverting the inspired and expired nasal cavity air currents which is expected to have more or less pressure stimuli on the nasal mucosal neuro receptors and on olfactory receptors. The acuity of the receptors' function is thought to play major rule on the power steering of the nasal breathing and smelling functions. Little correspondent research is found on that field owing to the lack of objective measurements.

\section{Surgical technique}

\section{Posterior septal graft by endoscopic resection of Area 4}

The majority of the difficult twisted noses are resulted from a previous septoplasty, rhinoseptoplasty or nasal injury. Reinstallations of Area $1 \& 3$ by a graft augmentation are essential. The frontocephalic part of Area 4 is usually inaccessible and often spared during nasal septal surgeries. The harvesting of the graft is carried out endoscopically under direct vision through a hemitransfixion incision alongside the M.Cottle line. A heavy scissors is used for separating the cephalic part from its attachment from the cribriform ethmoidal roof back to the sphenoidal rostrum. All anatomical structures within Area 4 are targeted regardless of its origin. It is mainly an ethmoidal perpendicular plate. My target is to remove $3 \times 2 \mathrm{~cm}$ graft.

The graft is shaped to simulate Area $1 \& 3$ and applied to the already existed deformed septal cartilaginous structure anterior to M.Cottle line. It is essential to stabilise and/or anchor the Area 3 of the graft to the midline of the external nasal bony structure in the form similar to the extended spreader graft. Simple suture to the anterior nasal spine is usually enough to guide the hard septal graft to the midline.

In case of not having enough cartilage anterior to the M.Cottle line, an auricular conchalcartilage graft is harvested and deployed.

I never required a drill assistance to create hols in the bony graft. I always used a thick green syringe needle to create hols in the bony graft before suturing it in place.

\section{Auto spreader upper alar cartilage graft}

Figure 3, the forthcoming technique has not been described or previously published. I use minimally two sutures to inward curving the free dorsal edges of the upper alar cartilages and securing them lateral to the dorsal edge of Area 3. The technique is ideal for reduction rhinoplasty and whenever the dorsal edges of the upper alar cartilages are higher than the neomiddle third of the nasal dorsum. It aims to keep the strength and the concavity "out bulging" of the upper alar septal junction by avoidance of stripping the mucosa off the inner aspect of the excessive dorsal edge of the upper alar cartilage and by avoidance of suturing the folded free edge on itself before tucking it lateral to the dorsal part of Area 3 as previously described.

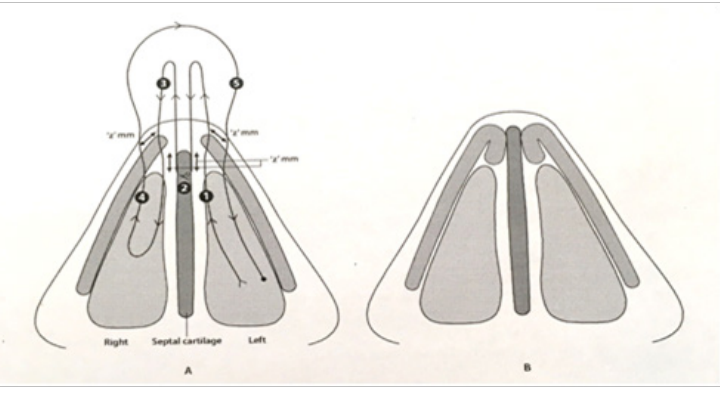

Figure 3 Modified Auto Spreader Graft.

\section{Technique}

Open rhinoplasty approach, SMAS nasal envelop is dissected. Trascolumellarbilateral mucoperichondral septal flaps are raised, the upper alar cartilages are submucosally dissected and separated off their septal cartilage attachment and the free edges of the upper alar cartilages are kept attached to the already elevated adjacent septal mucoperichodrium on both sides of Area 3 of the septal cartilage. The middle third of the new nasal septal dorsum is expected to be lower than the dorsal hight of the upper alar cartilages, the extra length should be estimated as a length figure " $\mathrm{z}$ " $\mathrm{mm}$. 5/0 PDS blue in colour and rounded body needle is used. The start suture is passed into the left nasal cavity through the left nostril out to the exposed nasal dorsum through the free margin of the left upper alar cartilage at its junction with the already dissected nasal septal mucoperichondrium. 
The second pass of the needle is through the already dissected nasal septal dorsal cartilage " $z$ "mm length deeper to the dorsal free edge. The third pass of the needle is through the free border of the right upper alar cartilage opposite to the same level of the left one, but in opposite direction from outside through the exposed nasal dorsum into the right nasal cavity, the needle is looked at through the right nasal cavity and pulled out of the right nostril. the fourth pass of the needle is from inside of the right nasal cavity through the body of the right upper alar cartilage " $\mathrm{z}$ " $\mathrm{mm}$ distance lateral to the entrance of the third pass, the needle is seen emerging into the exposed outer surface of the middle third of the nose. The fifth and last needle pass is " $\mathrm{z}$ " $\mathrm{mm}$ distance lateral to the first pass and through the body of the left upper alar cartilage, into the left nasal cavity where the other end of the thread is sitting, the needle is grasped and taken out of the left nasal cavity. The two ends of the thread are tied together with the help of the surgeon's index finger which is used for pushing them after they are winded on each other through the left nostril, the surgeon is kept watching the exposed nasal dorsum all the way to ensure inner curving of the excessive upper alar cartilage free edges lateral to the nasal dorsal septal cartilage on both sides as an upper alar cartilage auto spreader grafts.

Two to three sutures are applied.

\section{Advantages of the upper alar cartilage auto spreader graft new technique:}

A. It respects the key stone midline junction and maintain good pushing bulge of cartilage and gap filling bulge to that such risky area where a previous surgery or odd trauma was implicated. The spring type of curved cartilage gives a flexible out pushing contour which has a benefit on moulding the upper alar cartilage to its nasal bone attachment and on creating an open angulation with the septum.

B. It help on reduces the possible long term risk of developing an inverted $\mathrm{V}$ deformity on the side wall of the bony cartilaginous junction by respecting the natural lateral convexity of the cephalic upper alar cartilage and adding a lateral spring like action to the key stone cephalic attachment of the upper alar cartilage to the under surface of the nasal bone, the lateral aspect of the anterior nasal valve will be more splinted to the lateral bony attachment. This is also expected to maximise the opening of the angle with the medial boundary.

C. it minimises the surgical manipulation and trauma on the whole body of the upper alar cartilage.

\section{Disadvantages of the upper alar cartilage auto spreader graft new technique}

1. Broad and bulgy middle third appearance: It could be noticed for the first few weeks following the removal of the external nasal splints but never reported or noticed for after. The weight of the skin envelop and the healing scars including the contraction are expected to remould the possible bulge of the spring shape folded UAC upper alar cartilage.
2. Difficult to apply especially in case when dealing with strong excessive cartilage or feeling the necessity to apply one of the sutures very close to the cephalic end. I use 4/0 PDS rounded body in the first case and endoscopic assistance in the second case.

3. Cannot revise or reduce the hight of the middle third of the nasal dorsal when the sutures are applied. There is no way to suture the upper alar cartilage perichondrium on each other above the dorsal septum in this technique.

4. 4-The PDS suture nodes could be felt by the patient at introducing the little finger inside the nose. This is possible for the first three months and/or could track an infection. It is a theoretical risk, hasn't been seen over the last 100 cases of my work.

\section{Advantages of area 4 septal graft}

a. It is reliable and adequate. It is a good option in case of not having enough septal cartilage graft such as post reduction septoplasty, 2 The bony septal graft spares chest incision and is an alternative to the rib cartilage in some cases. It is stable, does not warp and could be used in conjunction with the conchal cartilage graft.

b. 3- Tongue-in-groove technique is applicable on the graft. It helps on keeping the nasal tip on the middle.

\section{Disadvantages of area 4 septal cartilage graft}

a. The bony-bony and bony-cartilage junctions of the graft are delicate and variable in thickness.

b. Careful endoscopic harvesting is essential. It requires decent knowledge about anterior skull base structures. It puts the patient through a potential risk of breaching the roof of the nasal cavity.

c. The graft requires creating needle holes before installation and could need a power drill.

d. It is sharp and dorsal masking cover is advisable in case of thin skin envelop.

\section{Acknowledgments}

None.

\section{Conflicts of interest}

Author declares there are no conflicts of interest.

\section{Funding \\ None.}

